\title{
An Average Cramer-Rao Bound for Frequency Offset Estimation in Frequency-Selective Fading Channels
}

\author{
Yinghui Li, Hlaing Minn, Senior Member, IEEE, and Jianqiang Zeng, Student Member, IEEE
}

\begin{abstract}
Several variations of Cramer-Rao bounds for carrier frequency offset (CFO) estimation in frequency-selective fading channels have been used to benchmark practical estimators' performance or to design training signals for CFO estimation. Among them, the extended Miller-Chang bound (EMCB) provides a tighter bound than the CRB for locally unbiased estimators. However, there is no closed-form expression of the EMCB for the CFO estimation in frequency-selective fading channels with an arbitrary training signal. In this letter, we derive a closed-form exact average CRB (the EMCB) valid for any training signal and any signal structure for the CFO estimation over frequency-selective Rayleigh fading channels with uncorrelated or arbitrarily correlated taps. The accuracy and generality of the proposed average CRB expression, and its advantages over the existing expressions are corroborated by numerical and simulation results.
\end{abstract}

Index Terms-Cramer-Rao bound (CRB), frequency offset estimation, frequency-selective fading, Rayleigh fading.

\section{INTRODUCTION}

$\mathbf{T}$ HE carrier frequency offset (CFO) between the transmitter and receiver can cause a severe performance degradation [1], [2], and hence several CFO estimators, e.g., [3]-[6], have been proposed in the literature. Due to the presence of other unknown parameters (e.g., channel impulse response, phase offset), the existing CFO estimators can be categorized into two types - a direct CFO estimation, e.g., [3], [5], and a joint estimation of $\mathrm{CFO}$ and channel impulse response (or generalized maximum likelihood approach), e.g., [4], [7][10]. To benchmark performance of these estimators, several variations of Cramer-Rao bound were derived in [4], [11][16]. Among them, the extended Miller-Chang bound (EMCB) provides a tighter bound than the $\mathrm{CRB}$, the hybrid $\mathrm{CRB}$, the modified CRB (MCRB), Miller-Chang bound, and asymptotic CRB for locally unbiased estimators ${ }^{1}$ [13]. When considering a joint estimation of $\mathrm{CFO}$ and channel impulse response, the corresponding $\mathrm{CRBs}$ of the $\mathrm{CFO}$ and the channel response only concern with the particular realization of channel and CFO. In particular, the CRB of CFO estimation in this case depends on the particular channel realization ${ }^{2}$ (and hence we

Manuscript received May 4, 2008; revised August 22, 2008 and November 15, 2008; accepted November 27, 2008. The associate editor coordinating the review of this letter and approving it for publication was Y. J. Zhang.

Y. Li is with the LitePoint Corp. (e-mail: yinghui.li@litepoint.com).

H. Minn and J. Zeng are with the Department of Electrical Engineering, University of Texas at Dallas (UTD) (e-mail: \{jianqiang.zeng, hlaing.minn\}@utdallas.edu).

This work was supported in part by the Erik Jonsson School Research Excellence Initiative, the University of Texas at Dallas.

Digital Object Identifier 10.1109/TWC.2010.03.080608

${ }^{1}$ those estimators that are unbiased for all values of the nuisance parameters.

${ }^{2}$ In some particular cases, a specifically designed training sequence may make the CRB decoupled from the channel realization [17] but the CRB will still depend on the energy of that channel realization, and hence an averaging over the random snap-shot channel energy would still be required. will call it a snap-shot CRB). If an average performance bound of CFO based on the joint estimation is desired, the above snap-shot CRB needs to be averaged over the channel fading. This average CRB corresponds to the extended Miller-Chang bound, and the Monte Carlo simulation is used to compute this average CRB (e.g., see [13] and [6]) as no closed-form expression is available.

The CRB or similar bounds are also useful in designing training signals [7], [8], [16], [18], but not all of them are analytically tractable. The use of average CRB in [8] provides better training signals than the use of asymptotic CRB in [7]. The average CRB is derived by means of an approximation in [8] for periodic training signals with cyclic prefix (CP) structure. The existing closed-form average CRB expression in [8] holds for neither an arbitrary training signal nor a zeropadded (ZP) signal structure. To the best of our knowledge, the closed-form exact average CRB expression for the CFO estimation in frequency-selective Rayleigh fading channels for an arbitrary training signal with any structure (CP or ZP) has not been derived in the literature, and is what we propose in this letter.

The rest of this paper is organized as follows. Section II describes the signal model, the CRB, the MCRB, and the snap-shot CRB. Section III derives the closed-form exact average CRB for an arbitrary training signal. Section IV provides performance comparison and discussions, and Section $\mathrm{V}$ concludes the paper. We use the following notations. The superscripts $T$ and $H$ denote the transpose and the Hermitian transpose, while $\operatorname{tr}(\cdot)$ stands for the trace operation. $\operatorname{diag}\left\{x_{0}\right.$, $\left.x_{1}, \ldots, x_{K-1}\right\}$ represents a $K \times K$ diagonal matrix whose diagonal elements are given by $x_{0}, x_{1}, \ldots, x_{K-1} . E[\cdot]$ stands for the expectation while $E_{\boldsymbol{x}}[\cdot]$ denotes the expectation over the random vector $\boldsymbol{x} . \boldsymbol{I}_{K}$ is the $K \times K$ identity matrix.

\section{Signal Model And THE SNAP-SHOT CRB}

Let us consider a wide-sense-stationary quasi-static frequency-selective Rayleigh fading channel characterized by its low-pass-equivalent channel impulse response (CIR) vector $\boldsymbol{h}=[h(0), h(1), \ldots, h(L-1)]^{T}$, where $L$ is the number of the sample-spaced ( $T_{s}$-spaced) CIR samples. $\boldsymbol{h}$ is a zero-mean complex Gaussian random vector with a covariance matrix given by

$$
\begin{aligned}
\boldsymbol{C}_{h} & =E\left[\boldsymbol{h} \boldsymbol{h}^{H}\right] \\
& =\boldsymbol{U}_{h} \operatorname{diag}\left\{\lambda_{h}(0), \lambda_{h}(1), \ldots, \lambda_{h}\left(L^{\prime}-1\right), 0, \ldots, 0\right\} \boldsymbol{U}_{h}^{H} \\
& =\tilde{\boldsymbol{U}}_{h} \tilde{\boldsymbol{\Sigma}}_{h} \tilde{\boldsymbol{U}}_{h}^{H}
\end{aligned}
$$

where $\tilde{\boldsymbol{\Sigma}}_{h}$ is the $L \times L$ diagonal matrix with non-increasing eigen-values of $\boldsymbol{C}_{h}, L^{\prime}$ is the number of nonzero eigen-values 
$\left\{\lambda_{h}(k)\right\}$ of $\boldsymbol{C}_{h}$, and $\tilde{\boldsymbol{U}}_{h}$ is constituted by the first $L^{\prime}$ columns of the unitary matrix $\boldsymbol{U}_{h}$. Define $v$ as the carrier frequency offset normalized by $1 /\left(N T_{s}\right)$ where $N$ is the number of training signal samples excluding the guard samples. Consider a transmitted training sequence $\{s(n): n=-L+1,-L+$ $\left.2, \ldots, 0,1, \ldots, N^{\prime}-1\right\}$. For CP based training structures, $N^{\prime}=N$, and $\{s(n): n=-L+1,-L+2, \ldots,-1\}$ are the $L-1 \mathrm{CP}$ samples. For ZP based training structures[19], $N^{\prime}=N+L-1$, and $\{s(n): n=-L+1,-L+2, \ldots,-1\}$ and $\{s(n): n=N, N+2, \ldots, N+L-2\}$ are the null prefix ${ }^{3}$ samples of the training signal and those of the following data signal, respectively. Then the received signal vector can be expressed in matrix form as

$$
\boldsymbol{r}=\boldsymbol{\Omega}(v) \boldsymbol{S h}+\boldsymbol{n}
$$

where $\boldsymbol{r}=\left[r(0), \quad r(1), \quad \ldots, \quad r\left(N^{\prime}-1\right)\right]^{T}, \quad \boldsymbol{h}=$ $[h(0), h(1), \ldots, h(L-1)]^{T}, \quad \boldsymbol{n}=[n(0), n(1), \ldots$, $\left.n\left(N^{\prime}-1\right)\right]^{T}, \boldsymbol{\Omega}(v)=\operatorname{diag}\left\{1, e^{j 2 \pi v / N}, \ldots, e^{j 2 \pi\left(N^{\prime}-1\right) v / N}\right\}$, $[\boldsymbol{S}]_{k, l}=s(k-l)$ for $0 \leq k \leq N^{\prime}-1,0 \leq l \leq L-1$, and $\boldsymbol{n}$ is the zero-mean white complex Gaussian noise vector with the covariance matrix $\sigma_{n}^{2} \boldsymbol{I}_{N^{\prime}}$.

For Rayleigh fading channels, $p(\boldsymbol{r} \mid v)$, the probability density function (pdf) of $\boldsymbol{r}$ given $v$ is zero-mean complex Gaussian with the covariance matrix

$$
\boldsymbol{C}_{r}=\boldsymbol{\Omega}(v) \boldsymbol{S} \boldsymbol{C}_{h} \boldsymbol{S}^{H} \boldsymbol{\Omega}^{H}(v)+\sigma_{n}^{2} \boldsymbol{I}_{N^{\prime}}
$$

and the $\mathrm{CRB}$ of the direct $\mathrm{CFO}$ estimation can be obtained as [14]

$$
\mathrm{CRB}=\frac{N^{\prime 2}}{8 \pi^{2}\left(\operatorname{tr}\left(\boldsymbol{R}^{-1} \boldsymbol{\Lambda} \boldsymbol{R} \boldsymbol{\Lambda}\right)-\operatorname{tr}\left(\boldsymbol{\Lambda}^{2}\right)\right)}
$$

where $\boldsymbol{R}=\boldsymbol{S}_{h} \boldsymbol{S}^{H}+\sigma_{n}^{2} \boldsymbol{I}_{N^{\prime}}$ and $\boldsymbol{\Lambda}=\operatorname{diag}\left\{0,1, \ldots, N^{\prime}-\right.$ $1\}$. The corresponding maximum likelihood estimator ${ }^{4}$ (MLE) of $v$ which maximizes $p(\boldsymbol{r} \mid v)$ is also known as Bayesian MLE and it requires the knowledge of $\boldsymbol{C}_{\boldsymbol{h}}$ and $\sigma_{n}^{2}$, and hence it is unrealizable for practical systems with unknown $\boldsymbol{C}_{\boldsymbol{h}}$ and $\sigma_{n}^{2}$. On the other hand, both $v$ and $\boldsymbol{h}$ can be jointly estimated based on ML principle as in [4], [7]-[10].

The (snap-shot) CRB for the $v$ derived from the joint estimation of $v$ and $\boldsymbol{h}$ is given by [4]

$$
\left.\mathrm{CRB}\right|_{\boldsymbol{h}}=\frac{\sigma_{n}^{2} N^{\prime 2}}{8 \pi^{2} \boldsymbol{h}^{H} \boldsymbol{S}^{H} \boldsymbol{\Lambda}\left(\boldsymbol{I}_{N^{\prime}}-\boldsymbol{B}\right) \boldsymbol{\Lambda} \boldsymbol{S} \boldsymbol{h}}
$$$$
\text { where } \quad \boldsymbol{B}=\boldsymbol{S}\left(\boldsymbol{S}^{H} \boldsymbol{S}\right)^{-1} \boldsymbol{S}^{H} .
$$

The notation CRB $\left.\right|_{\boldsymbol{h}}$ is used to reflect that the CRB is just for a given channel realization $\boldsymbol{h}$.

The MCRB is given by [11][13]

$$
\mathrm{MCRB}=\frac{\sigma_{n}^{2} N^{\prime 2}}{8 \pi^{2} \operatorname{tr}\left(\boldsymbol{S}^{H} \boldsymbol{\Lambda}\left(\boldsymbol{I}_{N^{\prime}}-\boldsymbol{B}\right) \boldsymbol{\Lambda} \boldsymbol{S} \boldsymbol{C}_{h}\right)} .
$$

\section{Average CRB}

In this section, we present the CRB of the CFO estimation based on the ML joint CFO and channel estimation in multipath Rayleigh fading channels. We derive the closed-form

\footnotetext{
${ }^{3}$ They can also be in the form of null suffix.

${ }^{4}$ which is given as $\hat{v}_{\mathrm{ML}}=\arg _{v} \min \left\{\boldsymbol{r}^{H} \boldsymbol{\Omega}(v) \boldsymbol{R}^{-1} \boldsymbol{\Omega}^{H}(v) \boldsymbol{r}\right\}$.
}

expression of the CRB averaged over a multipath Rayleigh fading channel as

$$
\overline{\mathrm{CRB}}=E_{\boldsymbol{h}}\left[\left.\mathrm{CRB}\right|_{\boldsymbol{h}}\right] .
$$

With the definition of

$$
Z=\boldsymbol{h}^{H} \boldsymbol{S}^{H} \boldsymbol{\Lambda}\left(\boldsymbol{I}_{N^{\prime}}-\boldsymbol{B}\right) \boldsymbol{\Lambda} \boldsymbol{S h},
$$

the snap-shot CRB can be expressed as

$$
\left.\mathrm{CRB}\right|_{h}=\frac{\alpha}{Z}=\left.\mathrm{CRB}\right|_{Z}
$$

where $\alpha=\frac{N^{\prime 2} \sigma_{n}^{2}}{8 \pi^{2}}$, and the average CRB can be obtained as $E_{Z}\left[\left.\mathrm{CRB}\right|_{Z}\right]$. Define

$$
\begin{aligned}
\tilde{\boldsymbol{h}} & =\tilde{\boldsymbol{\Sigma}}_{h}^{-1 / 2} \tilde{\boldsymbol{U}}_{h}^{H} \boldsymbol{h}, \\
\boldsymbol{Q} & =\tilde{\boldsymbol{\Sigma}}_{h}^{1 / 2} \tilde{\boldsymbol{U}}_{h}^{H} \boldsymbol{S}^{H} \boldsymbol{\Lambda}\left(\boldsymbol{I}_{N^{\prime}}-\boldsymbol{B}\right) \boldsymbol{\Lambda} \boldsymbol{S} \tilde{\boldsymbol{U}}_{h} \tilde{\boldsymbol{\Sigma}}_{h}^{1 / 2}=\boldsymbol{U} \boldsymbol{\Sigma}_{Q} \boldsymbol{U}^{H}
\end{aligned}
$$

where $\boldsymbol{U}$ is a unitary matrix and $\boldsymbol{\Sigma}_{Q}=$ $\operatorname{diag}\left\{\lambda_{0}, \lambda_{1}, \cdots, \lambda_{K-1}, 0, \ldots, 0\right\}$. Then (9) can be written as

$$
Z=\tilde{\boldsymbol{h}}^{H} \boldsymbol{Q} \tilde{\boldsymbol{h}}=\boldsymbol{g}^{H} \boldsymbol{\Sigma}_{Q} \boldsymbol{g}=\sum_{k=0}^{K-1} \lambda_{k}|g(k)|^{2}
$$

where $\boldsymbol{g}=\boldsymbol{U}^{H} \tilde{\boldsymbol{h}}$. In a multipath Rayleigh fading channel, both $\tilde{\boldsymbol{h}}$ and $\boldsymbol{g}$ are zero-mean complex Gaussian random vectors with the covariance matrix $\boldsymbol{I}_{L^{\prime}}$, and $Z$ is a sum of weighted Chi-square random variables.

Depending on the eigen-values of $\boldsymbol{Q}$, we divide the whole space of possible training signals into two cases:

1) Case I: $Q$ has at least two distinctive eigen-values.

The moment generating function of $Z$ in (13) is given by[20]

$$
\Phi_{Z}(s)=\int_{0}^{\infty} p_{Z}(z) e^{s z} d z=\prod_{l=1}^{m}\left(1-\lambda_{l} s\right)^{-\kappa_{l}}
$$

where $m$ is the number of nonzero distinct eigen-values $\left\{\lambda_{l}\right\}$ of $Q$ and $\kappa_{l}$ is the multiplicity of $\lambda_{l}$. By the inverse Laplace transform, the pdf of $Z$ can be expressed as [21]

$$
p_{Z}(z)=\sum_{l=1}^{m} \sum_{k=1}^{\kappa_{l}} A_{l k} \frac{1}{\lambda_{l}} f_{2 k}\left(z / \lambda_{l}\right)
$$

where $f_{n}()$ is a Chi-square pdf with $n$ degrees of freedom (DOFs), and

$$
\begin{aligned}
& f_{n}(x)=\frac{x^{n / 2-1} e^{-x}}{\Gamma(n / 2)}, 0<x<\infty \\
& A_{l k}=\left\{\prod_{i=1}^{m}\left(-2 \lambda_{i}\right)^{-\kappa_{i}}\right\}\left(-2 \lambda_{l}\right)^{k} b_{l k} \\
& b_{l k}=\left\{\sum_{j_{1}=0}^{\kappa_{l}-k-1}\left(\begin{array}{c}
\kappa_{l}-k-1 \\
j_{1}
\end{array}\right) A_{l}^{\left(\kappa_{l}-k-1-j_{1}\right)}\right. \\
& \times \sum_{j_{2}=0}^{j_{1}-1}\left(\begin{array}{c}
j_{1}-1 \\
j_{2}
\end{array}\right) A_{l}^{j_{1}-1-j_{2}} \\
& \left.\times \sum_{j_{3}=0}^{j_{2}-1}\left(\begin{array}{c}
j_{2}-1 \\
j_{3}
\end{array}\right) A_{l}^{j_{2}-1-j_{3}} \ldots\right\} \times \frac{\triangle_{l}}{\left(\kappa_{l}-k\right) !}
\end{aligned}
$$




$$
\begin{aligned}
\triangle_{l} & =\prod_{k=1, k \neq l}^{m}\left(\frac{1}{2 \lambda_{l}}-\frac{1}{2 \lambda_{k}}\right)^{-\kappa_{k}} \\
A_{l}^{(n)} & =(-1)^{(n+1)} n ! \times \sum_{k=1, k \neq l}^{m} \kappa_{k}\left(\frac{1}{2 \lambda_{l}}-\frac{1}{2 \lambda_{k}}\right)^{-(n+1)} .
\end{aligned}
$$

After some manipulation, the average CRB is given by

$$
\begin{aligned}
\overline{\mathrm{CRB}} & =\lim _{\beta \rightarrow 0} \int_{\beta}^{\infty} \frac{\alpha}{z} p_{Z}(z) d z \\
& =\alpha \lim _{\beta \rightarrow 0} \sum_{l=1}^{m}\left\{\frac{A_{l 1}}{\lambda_{l}} \mathrm{E}_{1}\left(\frac{\beta}{\lambda_{l}}\right)\right. \\
& \left.+\sum_{k=2}^{\kappa_{l}} \frac{A_{l k}}{(k-1) \lambda_{l}}\left(1-F_{2 k-2}\left(\beta / \lambda_{l}\right)\right)\right\}
\end{aligned}
$$

where $F_{n}(x)$ is the cumulative distribution function of a Chi-square random variable with $n$ DOFs, and $\mathrm{E}_{1}(x)$ is the exponential integral function defined by

$$
\mathrm{E}_{1}(x) \triangleq \int_{x}^{\infty} \frac{e^{-u}}{u} d u=-C-\ln (x)-\sum_{k=1}^{\infty} \frac{(-x)^{k}}{k \cdot k !}
$$

with $C$ being the Euler's constant. Based on (22), an approximate average $\mathrm{CRB}$ for periodic training signals with $\mathrm{CP}$ structure can be obtained as in [9]. In the following we will derive the closed-form exact average CRB expression for an arbitrary training signal with any structure $(\mathrm{CP}$ or $\mathrm{ZP})$.

According to the property of the Laplace transform, we have

$$
\lim _{z \rightarrow 0^{+}} p_{Z}(z)=\lim _{s \rightarrow \infty} s \Phi_{Z}(s) .
$$

From (15), the left side of (24) can be written as

$$
\lim _{z \rightarrow 0^{+}} p_{Z}(z)=\lim _{z \rightarrow 0^{+}} \sum_{l=1}^{m} \frac{A_{l 1}}{\lambda_{l}} e^{-z / \lambda_{l}}=\sum_{l=1}^{m} \frac{A_{l 1}}{\lambda_{l}} .
$$

Substituting (14) into the right side of (24), we have

$$
\lim _{s \rightarrow \infty} s \Phi_{Z}(s)=\lim _{s \rightarrow \infty} s \prod_{l=1}^{m}\left(1-\lambda_{l} s\right)^{-\kappa_{l}}=0, \quad m>1
$$

and from (24-26) we obtain

$$
\sum_{l=1}^{m} \frac{A_{l 1}}{\lambda_{l}}=0 .
$$

Substituting (27) and (23) back to (22), we obtain the average $\mathrm{CRB}$ as

$$
\begin{aligned}
\overline{\mathrm{CRB}} & =\alpha \lim _{\beta \rightarrow 0} \sum_{l=1}^{m}\left\{\frac{A_{l 1}}{\lambda_{l}}\left[-C-\ln \left(\frac{\beta}{\lambda_{l}}\right)-\sum_{k=1}^{\infty} \frac{\left(-\frac{\beta}{\lambda_{l}}\right)^{k}}{k \cdot k !}\right]\right. \\
& \left.+\sum_{k=2}^{\kappa_{l}} \frac{A_{l k}}{(k-1) \lambda_{l}}\left(1-\int_{0}^{\beta} f_{2 k-2}(u) d u\right)\right\} \\
& =\frac{N^{\prime 2} \sigma_{n}^{2}}{8 \pi^{2}} \sum_{l=1}^{m}\left\{\frac{A_{l 1}}{\lambda_{l}} \ln \lambda_{l}+\sum_{k=2}^{\kappa_{l}} \frac{A_{l k}}{(k-1) \lambda_{l}}\right\}
\end{aligned}
$$

2) Case II: $Q$ has $K$ identical eigen-values $\lambda$.

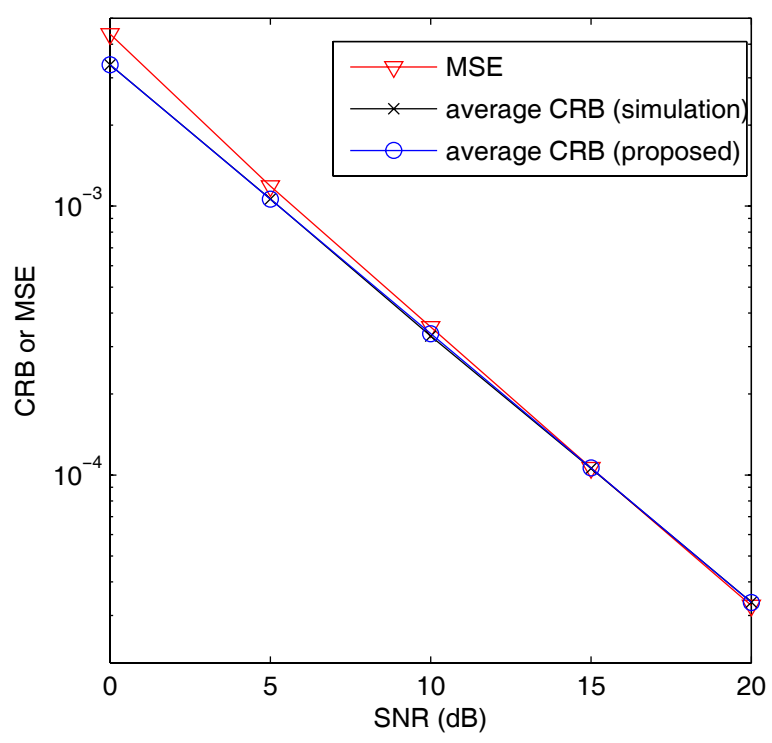

Fig. 1. The comparison of the proposed average CRB, simulated average $\mathrm{CRB}$ and the MSE for a CP-based training signal which yields all distinct eigen-values for $\boldsymbol{Q}$.

In this case, we have

$$
Z=\lambda \sum_{k=0}^{K-1}|g(k)|^{2} .
$$

Then, $Z$ is a Chi-square random variable with DOF $2 K$, and the pdf of $Z$ is given by

$$
p_{Z}(z)=\frac{z^{K-1}}{\Gamma(K) \lambda^{K}} e^{-z / \lambda}
$$

where $\Gamma(\cdot)$ is the gamma function. Substituting (30) into (21), we obtain the average CRB as

$$
\overline{\mathrm{CRB}}=\frac{N^{\prime 2} \sigma_{n}^{2}}{8 \pi^{2}} \frac{1}{(K-1) \lambda} .
$$

From (28) and (31), we observe that the average CRB is determined only by the eigen-values of $\boldsymbol{Q}$, which is closely related with the training signal matrix $S$ and the channel covariance matrix $C_{h}$. For a given $C_{h}$, the average CRB expressions (28) and (31) provide an easy computation of the exact average CRB for the CFO estimation in a frequencyselective Rayleigh fading channel for any training signal with any structure ( $\mathrm{CP}$ or $\mathrm{ZP})$. They also enable an easy numerical comparison of performance of different training signals.

\section{Performance Comparison}

We assume $N=64$ and $L=16$ in our simulation. We evaluate the CRBs in both uncorrelated and correlated multipath Rayleigh fading channels. The uncorrelated channel has 8 sample-spaced taps and an exponential power delay profile with $3 \mathrm{~dB}$ per tap decaying factor. The correlated sample-spaced channel is the Channel B of the indoor office ITU-R channel model[22].

We verify the accuracy of our closed-form exact average $\mathrm{CRB}$ expressions by comparing them with the Monte Carlo 


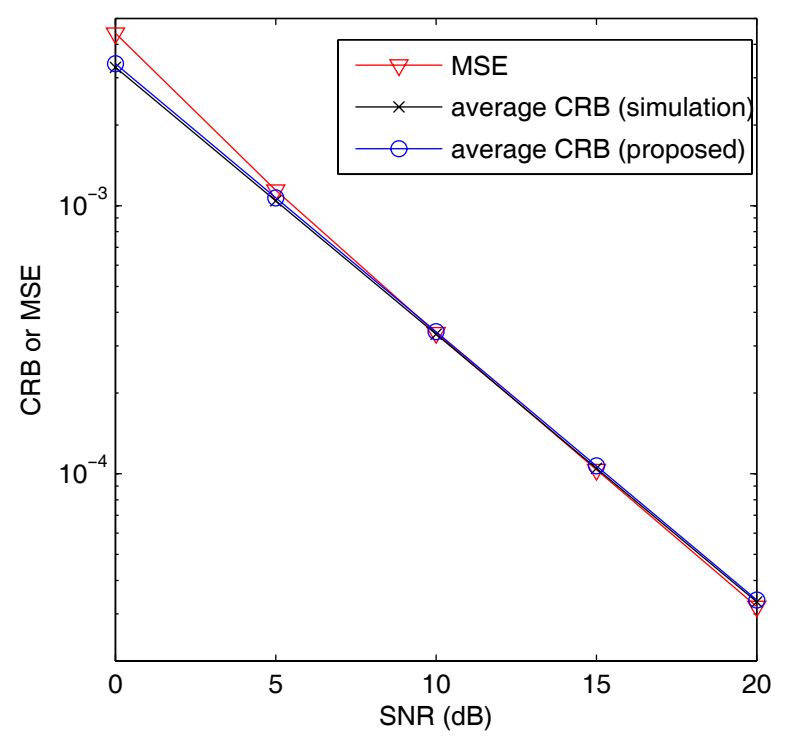

Fig. 2. The comparison of the proposed average CRB, the simulated average $\mathrm{CRB}$ and the simulated MSE for a CP-based training signal where three of the eigen-values of $\boldsymbol{Q}$ are identical.

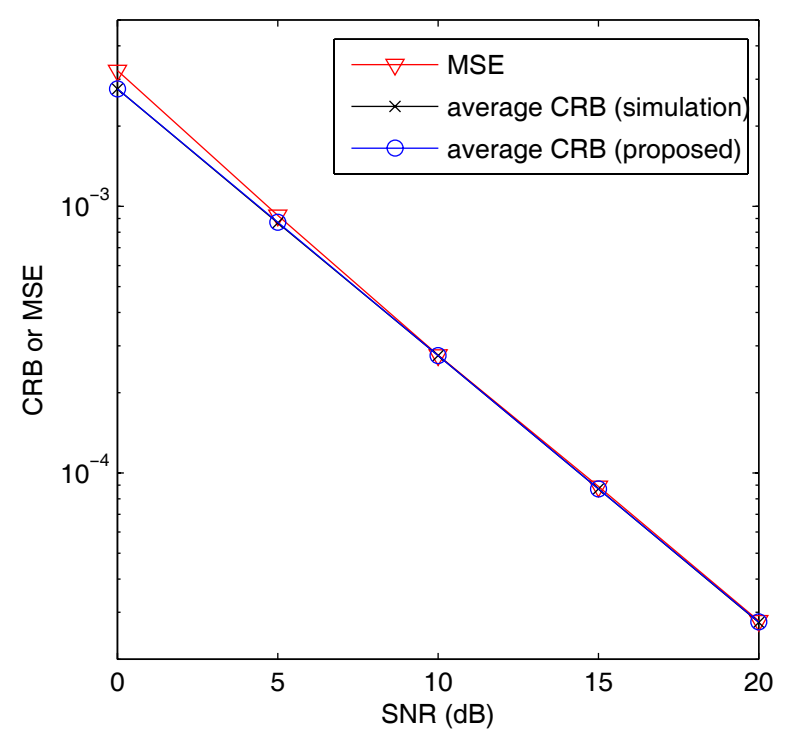

Fig. 3. The comparison of the proposed average CRB, simulated average $\mathrm{CRB}$ and the MSE for a CP-based training signal which yields all identical eigen-values for $\boldsymbol{Q}$.

simulation results of the average CRB for different nonperiodic training signals with $\mathrm{CP}$ structure in Figs. 1-3. Also included for the comparison are the mean-square error (MSE) simulation results of the generalized maximum likelihood CFO estimation proposed in [4]. Fig. 1 presents the results for the case where all eigen-values of $Q$ are distinct. Fig. 2 corresponds to the case where three of the eigen-values of $\boldsymbol{Q}$ are identical, while Fig. 3 shows the results for the case with all identical eigen-values of $\boldsymbol{Q}$. Our closed-form expressions exactly match with the Monte Carlo results for all the cases of the considered training signals. Note that the estimator's MSE closely follows the exact average CRB except at very (a) non-ZAC

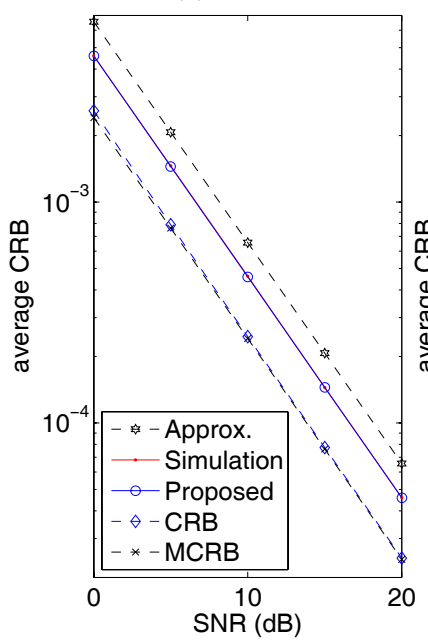

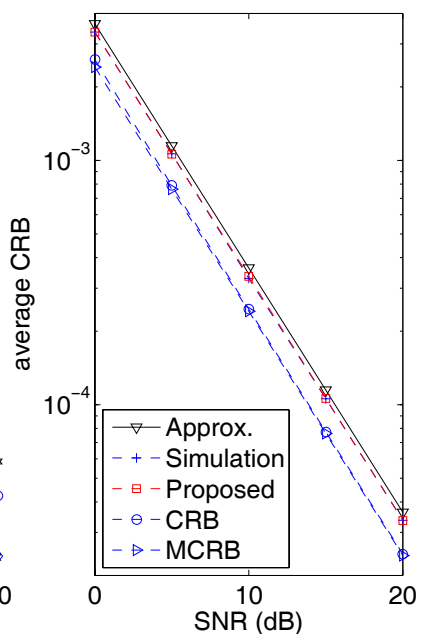

(b) CAZAC

Fig. 4. The comparison of the average CRBs obtained by simulation, by the proposed expression and by the existing approximate expression in [8], as well as the CRB and MCRB for different training sequences with ZP structure in uncorrelated multipath Rayleigh fading channels. (a) non-ZAC

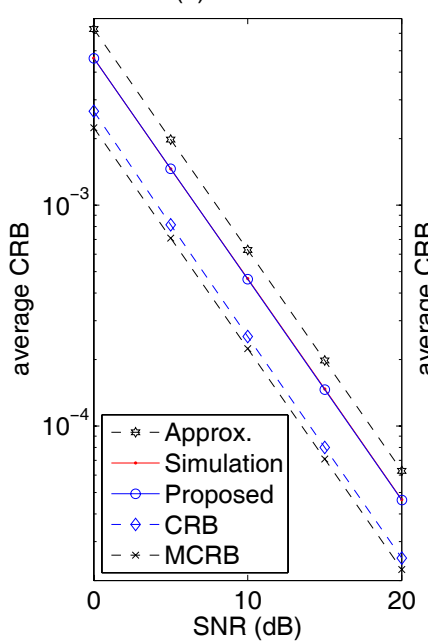

(b) CAZAC

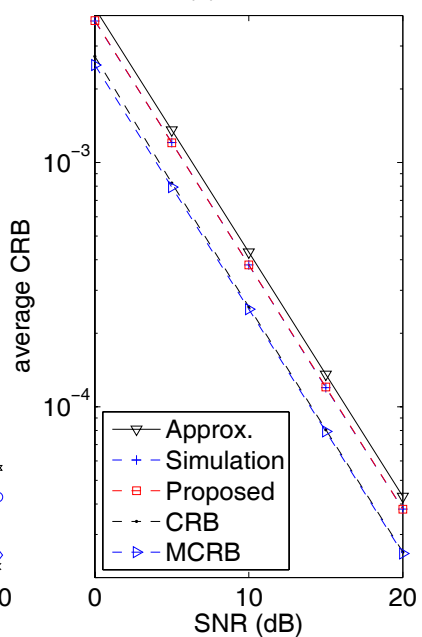

Fig. 5. The comparison of the average CRBs obtained by simulation, by the proposed expression and by the existing approximate expression in [8] as well as the $\mathrm{CRB}$ and the MCRB for different training sequences with $\mathrm{ZP}$ structure in correlated multipath ITU fading channels.

low SNR.

Fig. 4 and Fig. 5 plot the proposed exact average CRB, the existing approximate average CRB [8], the MCRB calculated by (7), the CRB calculated by (4), and the Monte Carlo results for several ZP periodic training signals [19] in multipath fading channels with uncorrelated taps and correlated taps, respectively.

The existing approximate average CRB expression yields slightly larger values than the Monte Carlo results for the constant amplitude zero auto-correlation (CAZAC) training sequence, and the performance gap becomes significantly larger for the non-ZAC training sequence. The gaps of the CRB and the MCRB to the Monte Carlo results are also large for both the CAZAC and non-ZAC training sequence, which indicates that $\mathrm{CRB}$ and the MCRB are rather loose 
although their computation may be simpler than our average CRB. It is interesting to note in Fig. 4 and Fig. 5 that the existing approximate average $\mathrm{CRB}$, which should represent a lower bound, may be even larger than the estimator's MSE, which underlines importance of the accurate average CRB. Our closed-form average CRB expression provides an exact match to the Monte Carlo MSE results for both correlated and uncorrelated multipath fading channels. These results highlight the accuracy and generality of our closed-form average CRB expressions and their advantages over the existing expressions. Note that the CFO estimation performance based on the ML joint CFO and channel estimation does not approach to the CRB (4) even for large SNR and training signal length. This is not contradictory to the well-known fact that MLE approaches to $\mathrm{CRB}$ asymptotically. The $\mathrm{CRB}$ in this case corresponds to the Bayesian MLE which requires the knowledge of the channel covariance matrix and the noise variance. Without those knowledge, the Bayesian MLE which maximizes $p(\boldsymbol{r} \mid v)$ cannot be realized. On the other hand, the ML joint CFO and channel estimation does not require those knowledge, and as illustrated in Figs. 1-3, its performance is matched by our average $\mathrm{CRB}$ expressions.

\section{COnClusions}

We have derived a closed-form exact average CRB (averaged over the channels) of the CFO estimation over multipath Rayleigh fading channels with uncorrelated or arbitrarily correlated taps for any training signal with any structure (CP or $\mathrm{ZP})$. In contrast to the limited applicability and/or inaccuracy of the existing average CRB expression, and the extensive Monte Carlo simulation run time required by other existing bounds, our closed-form expressions provide an easy computation of the exact average CRB without any limitation to the training signals and structures. Simulation results corroborate that our results closely match with the mean-square error performance of a practical generalized maximum likelihood estimator and are much more accurate than other existing bounds. Our average CRB expressions provide a useful metric for evaluating exact estimation performance bound in fading channels and for designing corresponding training signals.

\section{REFERENCES}

[1] T. Pollet, M. Van Bladel, and M. Moeneclaey, "BER sensitivity of OFDM systems to carrier frequency offset and Wiener phase noise," IEEE Trans. Commun., vol. 43, no. 234, pp. 191-193, 1995.
[2] H. Steendam and M. Moeneclaey, "Synchronization sensitivity of multicarrier systems," European Commun., vol. 52, pp. 834-844, May 2004.

[3] M. Morelli and U. Mengali, "An improved frequency offset estimator for OFDM applications," IEEE Commun. Lett., vol. 3, no. 3, pp. 75-77, 1999.

[4] —- "Carrier-frequency estimation for transmissions over selective channels," IEEE Trans. Commun., vol. 48, no. 9, pp. 1580-1589, 2000.

[5] H. Minn, P. Tarasak, and V. Bhargava, "OFDM frequency offset estimation based on BLUE principle," in Proc. IEEE Veh. Technol. Conf., vol. 2, Sept. 2002, pp. 1230-1234.

[6] D. Huang and K. Letaief, "Carrier frequency offset estimation for OFDM systems using null subcarriers," IEEE Trans. Commun., vol. 54, no. 5, pp. 813-823, 2006.

[7] P. Stoica and O. Besson, "Training sequence design for frequency offset and frequency-selective channel estimation," IEEE Trans. Commun., vol. 51, no. 11, pp. 1910-1917, 2003.

[8] H. Minn, X. Fu, and V. Bhargava, "Optimal periodic training signal for frequency offset estimation in frequency-selective fading channels," IEEE Trans. Commun., vol. 54, no. 6, pp. 1081-1096, 2006.

[9] X. Fu, X. Fu, H. Minn, and C. Cantrell, "Two novel iterative joint frequency-offset and channel estimation methods for OFDMA uplink," IEEE Trans. Commun., vol. 56, no. 3, pp. 474-484, 2008.

[10] Y. Li, H. Minn, N. Al-Dhahir, and A. R. Calderbank, "Pilot designs for consistent frequency-offset estimation in OFDM systems," IEEE Trans. Commun., vol. 55, no. 5, pp. 864-877, 2007.

[11] A. D'Andrea, U. Mengali, and R. Reggiannini, "The modified CramerRao bound and its application to synchronization problems," IEEE Trans. Commun., vol. 42, no. 234, pp. 1391-1399, 1994.

[12] F. Gini, R. Reggiannini, and U. Mengali, "The modified Cramer-Rao bound in vector parameter estimation," IEEE Trans. Commun., vol. 46, no. 1 , pp. 52-60, 1998.

[13] F. Gini and R. Reggiannini, "On the use of Cramer-Rao-like bounds in the presence of random nuisance parameters," IEEE Trans. Commun., vol. 48 , no. 12 , pp. $2120-2126,2000$.

[14] M. Ghogho, A. Swami, and T. Durrani, "Frequency estimation in the presence of Doppler spread: performance analysis," IEEE Trans. Signal Process., vol. 49, no. 4, pp. 777-789, 2001.

[15] G. Tavares, L. Tavares, and M. Piedade, "On the Miller-Chang lower bound for NDA carrier phase estimation," IEEE Trans. Commun., vol. 52, no. 11, pp. 1867-1871, 2004

[16] S. Sezginer and P. Bianchi, "Joint frequency offset and channel estimation in the OFDMA uplink: Cramer-Rao bounds and training sequence design," in Proc. IEEE 6th Workshop on Signal Processing Advances in Wireless Communications, 2005, pp. 585-589.

[17] M. Ghogho and A. Swami, "Training design for multipath channel and frequency-offset estimation in MIMO systems" IEEE Trans. Signal Process., vol. 54, no. 10, pp. 3957-3965, 2006.

[18] H. Minn and S. Xing, "An optimal training signal structure for frequency-offset estimation," IEEE Trans. Commun., vol. 53, no. 2, pp. $343-355,2005$.

[19] B. Muquet, Z. Wang, G. Giannakis, M. de Courville, and P. Duhamel, "Cyclic prefixing or zero padding for wireless multicarrier transmissions?" IEEE Trans. Commun., vol. 50, no. 12, pp. 2136-2148, 2002.

[20] A. Papoulis and S. U. Pillai, Probability, Random Variables and Stochastic Processes, 4th ed. McGraw-Hill, 2002.

[21] A. M. Mathai and S. B. Provost, Quadratic Forms in Random Variables: Theory and Applications. New York: Marcel Dekker, 1992.

[22] Recommendation ITU-R M.1225: Guidelines for evaluation of radio transmission technologies for IMT-2000, Std., 1997. 